\title{
La temática ambiental en la prensa escrita
}

\section{El caso de los periódicos de Saltillo, Coabuila}

\author{
JULIETA CARABAZA*
}

Resumen. Este trabajo analiza la cobertura ambiental que tres medios de comunicación de la ciudad de Saltillo, Coabuila: Vanguardia, El Diario de Coahuila $y$ Palabra, bicieron durante el primer trimestre de 2002. Es un análisis de contenido de notas informativas, reportajes, desplegados, editoriales $y$ secciones especializadas, realizado durante un periodo de 30 dias consecutivos.

El estudio tiene como punto de partida una pregunta central: ¿los temas ambientales forman parte de la agenda de los periódicos que circulan diariamente en el municipio de Saltillo, Coabuila?

El análisis de los datos ofrece algunos elementos interesantes. En primer lugar, la temática ambiental si es incluida en la prensa diaria, pero con un "bajo perfil", además, se evidencia que existe poco trabajo de reflexión o de investigación en el desarrollo de los tópicos que la conforman, que permita incidir en la construcción de una opinión püblica, cuya conciencia se encuentre más comprometida con el entorno natural. Por otro lado, su presencia está dada en función de los acontecimientos ambientales que se presentan de manera coyuntural en la sociedad o en función de las fuentes generadoras de la misma información, principalmente las gubernamentales.

Finalmente, se concluye que el análisis de la temática ambiental en la prensa, aunque no ofrece un panorama amplio sobre la problemática ambiental local, proporciona algunos indicadores importantes para quienes toman decisiones y diseñan estrategias para el mejoramiento de esta problemática biosocial.

-Especialista en comunicación masiva, comunicación educativa y estudios de recepción. Universidad Autónoma de Coahuila. Correo electrónico: jcaraba@mail.uadec.mx 


\section{Introducción}

as preocupaciones del hombre del siglo xxI
son muchas; entre ellas se encuentran, dice
Cienfuegos (2003), las que se refieren a los aspectos del desarrollo sustentable, la protección de la naturaleza y las reflexiones sobre el impacto del género humano en su entorno ambiental.

Ante esta problemática, la comunicación, entendida como un proceso cultural y dialéctico en donde los componentes del sistema al que se refiere el modelo: los actores, los instrumentos, las expresiones y las representaciones que se encuentran en relación con los objetos de referencia, las intervenciones y las mediaciones originadas en el sistema social (Serrano, 1993: 161 y 172), deben ser consideradas como un elemento fundamental para coadyuvar en el desarrollo de la cultura ambiental, principalmente en las sociedades modernas, donde los medios de comunicación (instrumentos) contribuyen de manera decisiva, junto con otras instituciones sociales, en la construcción de la representación de la realidad del hombre y su entorno.

Además, porque el éxito del movimiento ecologista, al menos a nivel mundial, dice Castells (2001: 153), obedece al hecho de que más que ninguna otra fuerza social ésta ha sido capaz de adaptarse lo mejor posible a las condiciones de la comunicación y al nuevo paradigma informacional a través de la utilización de los medios de comunicación para alcanzar audiencias más amplias y lograr así la legitimación de sus acciones.

Por lo cual, el presente trabajo explora, a partir de los principios teóricos de la Agenda Setting y mediante el análisis de contenido, los referentes que, sobre el asunto medioambiental, la prensa diaria de la ciudad de Saltillo, Coahuila puso a disposición de sus lectores durante el primer trimestre de 2002. Todo esto, con el fin de aportar elementos que permitan aproximarse a la comprensión sobre el papel que juega la prensa escrita local en la socialización de los temas medioambientalistas entre los habitantes de este municipio.

Los objetivos fundamentales se centran en: 1) determinar si son incluidos en la agenda de los medios impresos los temas relacionados con el medio ambiente; 2) determinar cuál/es es/son la/s temática/s ambiental/ es que se privilegian a través del periodismo local, y 3) contar con elementos que permitan aproximarse a entender la representación que hacen los periódicos desde sus propios referentes e intereses institucionales sobre la problemática del medio ambiente.

Lo anterior, considerando que para hablar de cultura ambiental en este siglo es importante comprender el papel que juegan los medios masivos de comunicación, ya que es a través de ellos desde donde se arman, en muchos de los casos, las discusiones de los asuntos globales, aunque también locales, de la problemática ambiental. 


\section{Fragmentos de algunos estudios sobre medios de comunicación y medio ambiente}

Aunque la preocupación de los problemas ambientales data, aproximadamente, de la segunda mitad del siglo pasado, el estudio del medio ambiente, desde la perspectiva social y especialmente la comunicacional, es un tema que ha tomado importantes dimensiones en la discusión académica, en un principio de modo intermitente, desde la década de los setenta en los Estados Unidos, y en los ochenta a nivel internacional (Dunlap, 1991 y 2002). Y es precisamente, en los ochenta cuando los medios de comunicación masiva -estimulados por los activistas del medio ambiente, los científicos y los elaboradores de políticas en los Estados Unidos- focalizaron su atención en gran medida sobre dichos problemas, por lo cual el tema adquirió dimensiones importantes en la opinión pública (Dunlap y Mitchell en Dunlap, 1991). Situación que no se ha visto reflejada en los medios mexicanos.

Por su parte, Hansen (1991) refiere que la investigación sobre el relato de la temática del medio ambiente en los medios de comunicación ha seguido los mismos pasos que cualquiera de los otros temas de la investigación en comunicación y medios, y que dicha semejanza incluye muchas de las mismas debilidades de la investigación en comunicación en general. Esto es, analizar los problemas ambientales desde la mirada de los procesos de comunicación a través de la transmisión lineal o de la información. Ante lo cual, el autor plantea que para entender el rol de los medios de comunicación en el desarrollo y elaboración de la temática del medio ambiente se debe trasladar la investigación hacia un marco constructivista en donde se reconozca la resonancia cultural sobre el privilegio de algunos temas sobre otros: esto es, adoptar una visión holística del rol de los medios en la construcción de los problemas sociales, especialmente en los referentes al medio ambiente, porque dice Hansen, el foco sobre los problemas sociales se encuentra encima de los mismos medios.

En México, los estudios acerca de la relación de los medios masivos de comunicación y el medio ambiente son aún muy escasos. Uno de los primeros trabajos elaborados de manera formal sobre el tema es el de Fregoso (1993), quien realizó un estudio sobre la cobertura que los diarios más importantes de Guadalajara, Jalisco, hicieron sobre el suceso del 22 de abril de 1992. Entre los hallazgos más importantes, el autor menciona que por el número de planas y superficie redaccional El Informador fue el periódico que presentó un mayor número de información; encontrando una serie de diferencias entre los distintos periódicos, las cuales se correlacionan con las características históricas y organizacionales de los mismos; en el plano cualitativo, los cuatro periódicos privilegiaron los aspectos descriptivos y de intervención, solamente dos periódicos aporta- 
ron elementos explicativos acerca del suceso; la categoría de contexto fue ignorada y solamente uno de ellos, Siglo 21, realizó el mejor trabajo de temporalización y $E l$ Occidental, trabajó más la hesitación. Finalmente, concluye el autor, la ausencia de ingredientes explicativos y contextuales puso de manifiesto el carácter poco indagatorio de la prensa tapatía.

Por su parte, Yescas Laguna (1999-2000) llevó a cabo una investigación que tituló La difusión ambiental a través de audiovisuales cuyo propósito principal fue realizar un análisis de contenido en dos guías televisivas para conocer la cantidad de programas que con contenido medioambiental se transmiten en las distintas televisoras nacionales de cobertura abierta (canales $4,5,7,9,11,13,22$ y 40 ).

De acuerdo a los resultados del análisis de los datos de este estudio, el investigador encontró que solamente $5.6 \%$ de los programas que se transmiten tienen el carácter de documentales, esto es, producciones de tipo didáctico-científico, y de los cuales sólo la mitad de ellos abordan la temática ambiental. Además, comenta el investigador, existen canales televisivos de la muestra estudiada que no transmiten ningún documental y los que lo hacen, lo realizan durante altas horas de la noche o muy temprano. El estudio arrojó que alrededor de $2 \%$ de los programas de contenido ambiental se transmiten en horario aceptable, lo cual, si se compara con el resto del contenido televisivo, el número de programas que se ponen para el disfrute $y$ aumento de la cultura ambiental es "insuficiente", dice el autor.

Recientemente Esteinou (2001) en el texto Ecología y medios de comunicación en México plantea, a partir de la reflexión de temas como: modernización, cultura y naturaleza, ecología, medios de comunicación y cambio de conciencia, y medios de comunicación y formación de una nueva cultura del agua en Baja California Sur, que:

[...] es urgente formar paralelamente a nivel global, vía los frentes culturales y los medios de comunicación colectivos, otro proceso mental superior que evite el avance de la cultura de la muerte [...] es necesario construir, a través de los principales medios de difusión y otras infraestructuras educativas, una globalización cultural y comunicativa, orientada hacia la defensa y conservación de la vida.

A partir de la síntesis de las investigaciones anteriores, se concluye que la tendencia de la investigación sobre los tópicos del medio ambiente desde la mirada de la comunicación en México es aún más esporádica y coyuntural que en otros países, como los Estados Unidos, por lo que es necesario seguir avanzando en esta línea, en principio, porque la problemática ambiental sigue creciendo y afectando los distintos ámbitos de la vida social, pero principalmente, porque en una sociedad como la actual, dominada por las representaciones que los medios de comunicación construyen del entorno de la misma, es necesario seguir dibujando el mapa 
que permita comprender el papel que juegan los medios ante problemáticas sociales como el deterioro ambiental.

\section{El enfoque teórico para el análisis de la prensa y el medio ambiente}

Para analizar la cobertura que hacen los medios masivos de comunicación sobre el tema del medio ambiente, se eligió la perspectiva teórica de la construcción del temario o Agenda Setting, teoría que proporciona los elementos que permiten reflexionar sobre cómo los medios de comunicación, a través de los diferentes géneros que manejan, contribuyen a construir día a día la compleja realidad de la sociedad actual.

Este enfoque que se inscribe en los estudios de los efectos a largo plazo, constituye una tendencia que ha fundamentado una gran cantidad de investigaciones desde su popularización en los años setenta, principalmente en aquéllas que abordan asuntos políticos como las campañas electorales (McCombs y Shaw, 1993). Sin embargo, su utilidad teórica y metodológica puede alcanzar otros ámbitos en donde los medios también tienen una responsabilidad social importante, como es el caso de la temática ambiental ya que la presencia pública sobre informaciones/ acontecimientos acerca del entorno, en este caso del medio ambiente, pueden influir y determinar el grado de atención que el público otorga a ciertos temas (Saperas, 1987).

La teoría de la Agenda Setting sostiene que los media son eficaces para construir la imagen de la realidad que el sujeto va estructurando y que esta construcción se suma a una metáfora de la totalidad de la información sobre el mundo que cada individuo organiza y almacena, la cual es comparada con la nueva información para así darle un significado (Wolf, 1991: 166).

La hipótesis central de la Agenda Setting ofrece tres niveles u objetos de estudio, como los llama Saperas (1987: 63-65): a) el nivel centrado en el "orden del día" de los temas, argumentos o problemas presentes en la agenda de los media, relacionado, además, con la jerarquía de importancia y la prioridad con la que dichos elementos son dispuestos en el orden del día; $b$ ) el nivel que se encarga de contrastar las agendas de los medios con la pública y que sirve para determinar el grado de influencia cognitiva que caracteriza a los medios; $c$ ) finalmente, el nivel de estudio que trata de explicar la relación de la naturaleza de los efectos y el marco temporal en el que se desarrollan acumulativamente. Niveles que permiten, en su conjunto, aproximarse a la comprensión del proceso cognitivo que los medios de comunicación producen en los diferentes públicos.

Así pues, se puede decir que esta perspectiva teórica ayuda a tener claro que los medios masivos de comunicación poseen influencia para 
determinar lo que es importante y relevante sobre los temas ambientales y pueden distinguirse los temas a los que se les da mayor importancia, issues, de los que no se les da tanta "centralidad".

Ante lo que se deberá tener presente que la tematización o construcción de la agenda en la producción periodística supone la selección de un tema y su colocación en el centro de la atención pública y sirve para que la opinión pública reduzca la complejidad social y focalice su atención en ciertos temas, lo que llevará a largo plazo a la construcción de la agenda pública, dependiendo, además, de las cargas cognitivas adquiridas en otros ámbitos sociales ya que "cuanto menor es la experiencia directa que tiene la gente sobre una determinada área temática, más depende de los media para obtener las informaciones y los marcos de interpretación correspondientes" (Zucker en Wolf, 1991: 175).

La selección del tema estará determinada por la interrelación de distintos factores como las mismas características del acontecimiento que decide la información periodística, relacionadas éstas con el grado de variación del sistema, la comunicabilidad del hecho y la implicación de los sujetos, tanto del destinatario como del mismo productor de la noticia, además de la relación acontecimiento-fuente-noticia, entre otros más (Rodrigo, 1989). Factores que, en algunos momentos, son utilizados para espectacularizar el acontecimiento, lo que trae como consecuencia que sólo unos cuantos sean "noticiables" (Wolf, 1991), en relación con la totalidad de los acontecimientos que se generan en la sociedad, lo que pone de manifiesto, por lo tanto, que los individuos que conforman dicha sociedad cuentan sólo con un conocimiento parcial del entorno que les rodea; pero además, que para cada uno de los periódicos la importancia de los acontecimientos será diferente y por lo tanto, su tratamiento también.

El término espectacularización indica la transformación y el tratamiento que sufren ciertos acontecimientos al momento de convertirlos en noticia con el fin de apelar a los sentimientos de los lectores, despertando el morbo o intentando producir sensaciones, emociones o impresiones con las noticias o sucesos que se ponen en circulación. Esto es, se juega con las reacciones del lector y se olvida cualquier parámetro de responsabilidad. Lo que arrastra, en muchos casos, a los sectores de la población más vulnerables a permanecer en un estado constante de mentira por la desproporción de la información o sometimiento a lo superficial.

Centrándonos pues, en este trabajo, es pertinente aclarar que éste se ubica en el primer nivel de análisis de la teoría, esto es, en la identificación de la composición y formación de la agenda sobre el tema ambiental. Así pues, se trata de tener claro los temas ambientales que son relevantes para los medios de comunicación de una ciudad media como Saltillo, de distinguir a los que se les da mayor importancia, issues, de los que no se les da tanta "centralidad" y, finalmente, de contar con elemen- 
tos básicos para acercarse a la representación que los mismos medios de comunicación tienen sobre el medio ambiente.

\section{Metodologia}

Con base en la perspectiva teórica de la Agenda Setting, para el análisis sobre los temas del medio ambiente que los periódicos Vanguardia, El Diario de Coabuila y Palabra que circulan en Saltillo, Coahuila, ponen a disposición de sus lectores, se plantearon las siguientes preguntas de investigación:

¿Cuáles son los temas ambientales que se emiten al público a través de cada uno de los periódicos locales? ¿a qué tipo de acontecimientos o actividades ambientales y con qué frecuencia le otorgan mayor énfasis los medios impresos locales? ¿qué temas ambientales se privilegian o minimizan en la información generada por los diarios de circulación local? ¿qué porcentaje de la información sobre el medio ambiente corresponde a temas de la localidad, del estado, o de la ciudad de México? ¿qué tanto acceso se le proporciona, a las fuentes oficiales ambientalistas en comparación con las de otros sectores de la población? ¿se encuentra balanceada la presencia de fuentes oficiales, locales, nacionales, iniciativa privada $u$ organizaciones civiles que se dedican a actividades a favor del medio ambiente en la prensa local?

Para responder a las preguntas de investigación planteadas en el presente trabajo y definir la tematización sobre el medio ambiente en la prensa de Saltillo, Coahuila, se usó la técnica del análisis de contenido porque, como afirma Lozano (1994) "es una técnica cuantitativa de investigación. útil para el estudio sistemático de los mensajes comunicacionales [...] y cumple los requisitos de sistematicidad y confiabilidad".

Este primer acercamiento a la conformación de la agenda que los medios impresos presentan sobre el tema del medio ambiente se realizó a partir de un seguimiento de prensa elaborado para el Instituto Estatal de Ecología durante 2002. Pero el presente reporte comprende sólo los primeros tres meses del año mencionado.

Las unidades de análisis definidas fueron: la nota informativa, reportajes, desplegados y secciones especializadas, que se encuentran en cualquiera de las páginas de los periódicos estudiados.

Los tópicos que conforman la temática ambiental para el análisis de la agenda de los periódicos se establecieron a partir de la Agenda 21 (Sato, 1997), en donde se proponen una serie de aspectos que deben ser incluidos y/o atendidos, a través de sistemas de gestión, en los diferentes niveles de organización social: 
Protección de la atmósfera

- Alternativas para combatir la contaminación atmosférica.

- Sistemas energéticos y nuevas tecnologías (energía solar, eólica y biomasa entre otras).

- Manejo ambiental junto con las comunidades, conciliando la protección del ambiente con el crecimiento económico.

- Desarrollo de programas de consumo energético eficiente para los usuarios.

- Programas educativos con relación al consumo energético.

Manejo del suelo

- Desarrollo de políticas del uso del suelo.

- Mejorar y hacer efectiva la legislación del uso del suelo.

- Empleo de técnicas como la planeación paisajista ecológica en las zonas rurales.

- Impulso de los diferentes estratos de la sociedad en los procesos de toma de decisiones.

- Orientación de las instituciones relacionadas con la cuestión del suelo a considerar la planeación con base en las dimensiones social y económica.

Combate a la deforestación

- Reforestación y control de incendios, plagas y contaminación perjudicial para los bosques.

- Incentivos para la investigación sobre plantas medicinales, ingeniería genética, fibras, resinas, entre otros.

- Utilización de métodos menos contaminantes para la expansión industrial.

- Política forestal.

- Incentivos para el turismo ecológico.

- Construcción de áreas verdes.

Combate a la desertificación y la sequía

- Planes de uso de manejo del suelo empleando agua potable.

- Programas preestablecidos para la reforestación y el manejo ambiental.

- Programas alternativos de energía y antidesertificación.

- Capacitación de la población para programas de irrigación, agrodesarrollo y conservación del agua. 
Desarrollo sustentable de las regiones montañosas

- Control de la erosión.

- Conservación de los recursos y empleo de tecnologías adecuadas para las regiones montañosas.

- Áreas de protección ambiental en las zonas montañosas.

Agricultura sustentable y desarrollo rural

- Conocimientos indígenas o campesinos para el control de las plagas.

- Uso de plaguicidas y productos químicos.

- Programas educativos y políticas de asentamientos humanos que favorezcan el empleo de tecnologías para los sistemas agrícolas.

Conservación de la biodiversidad

- Programas de manejo ambiental.

- Estrategias nacionales para la conservación de la biodiversidad.

- Inversión en investigación, educación, información y legislación.

- Impulso de métodos tradicionales de agricultura.

- Uso sustentable de tecnología.

- Creación de áreas de protección ecológicas de los sistemas dañados y la conservación de la biodiversidad.

Manejo de la biotecnología

- Cualidades nutricionales de los productos alimenticios.

- Desarrollo de vacunas para la prevención de enfermedades incluyendo los agentes patógenos en el agua potable.

- Control biológico de plagas.

- Promoción de la capacitación, investigación intercambio científico para el manejo de biotecnología.

Protección y manejo de los océanos

- Degradación de los mares.

- Aplicación de la legislación como medida contra la contaminación en relación con la descarga de nutrientes principalmente nitrógeno y fósforo causantes de la contaminación de los mares.

- Mejoramiento de la calidad de vida de los pescadores y su comunidad.

- Protección /manejo/conservación de los arrecifes, los acuarios, los pantanos y otras áreas oceánicas.

- Investigación de la capacidad de los soportes de los ecosistemas marinos.

- Contaminación de los mares. 
Protección y manejo del agua dulce

- Agua potable a todos los ciudadanos.

- Promoción de cursos de capacitación para el empleo del agua en los procesos de irrigación.

- Control y combate de las enfermedades transmitidas por el agua contaminada.

- Impulso a la investigación para el manejo de los ecosistemas acuáticos y agua potable.

- Tratamiento de las aguas negras.

Uso adecuado de los productos tóxicos

- Códigos internacionales de principios para prevenir y combatir los riesgos de impactos ambientales.

- Medidas de información y capacitación sobre emisiones tóxicas.

Manejo de residuos peligrosos

- Innovación industrial para combatir la producción excesiva de residuos peligrosos.

- Auditorias ambientales en las industrias.

- Programas de reciclaje y sustitución de materiales peligrosos.

- Programas de capacitación al público para reducir la producción de desechos peligrosos.

Manejo de residuos sólidos y líquidos

- Proceso de reciclaje.

- Nuevas alternativas energéticas.

- Aplicación de las leyes a quienes contaminen.

- Vigilancia de la industria.

- Mejorar los sistemas de irrigación.

- Establecimiento de patrones internacionales para el tratamiento y manejo de residuos sólidos.

- Tratamiento adecuado de los residuos.

Manejo de residuos radioactivos

- Formas para minimizar los desechos radioactivos, incluyendo transporte, procesamiento y acondicionamiento de forma segura.

- Respeto a la ley de frontera y no exportar los desechos radioactivos a otros países.

- Otros subtemas. 
Durante los tres meses de seguimiento diario a la prensa local se registraron 428 unidades que abordaban diferentes tópicos sobre el tema del medio ambiente. El total de unidades identificadas (notas informativas, reportajes, desplegados, secciones especializadas y editoriales) que sirvieron como base para el trabajo que hoy se presenta, constituían el $2 \%$ del total de la información manejada por los periódicos estudiados. Los diarios Vanguardia y Palabra concentraron respectivamente el $40 \%$ de las unidades analizadas, y el periódico El Diario de Coabuila, registró solamente el $20 \%$ (cuadro 1 ).

CUADRo 1. Distribución de medios estudiados

\begin{tabular}{ccc}
\hline Nombre del medio & num. de unidades & $\%$ \\
\hline Vanguardia & 172 & 40 \\
Palabra & 172 & 40 \\
El Diario & 84 & 20 \\
Total & 428 & 100 \\
\hline
\end{tabular}

Los datos contenidos en el cuadro 2 permiten afirmar que la nota informativa es el género más utilizado para abordar el tema del medio ambiente, situación que confirma lo que algunos estudiosos de la comunicación comentan con respecto a que la representación social de cualquier acontecimiento, hoy más que nunca, se pone a disposición del público en forma de noticia a la que se le define como una representación social de la realidad cotidiana producida institucionalmente ya que los objetos sociales que se visualizan a través de ella, no son objetos que se encuentren ya hechos en alguna parte de la realidad, sino que nos son dados a conocer por los medios a través de los distintos géneros periodísticos, por lo que se ha llegado a afirmar que los acontecimientos existen en la medida la en que son elaborados por los mismos medios de comunicación, tal como puede ser el caso de la temática ambiental (Rodrigo, 1998: 185).

Asimismo, es importante resaltar que se encontró información en forma de reportaje y editorial de manera esporádica (cuadro 2). Además, se evidencian diferencias en el uso de estos géneros de acuerdo a los periódicos. Así, Palabra y El Diario fueron los medios impresos que utilizaron un poco más el reportaje para comunicar a sus lectores los asuntos medioambientales de la localidad; y el editorial, a su vez, fue utilizado por los periódicos Vanguardia y Palabra (cuadro 3). 
CuADro 2. Distribución de las unidades de análisis

\begin{tabular}{lcc}
\hline Tipo de unidad & Nüm. de unidades & $\%$ \\
\hline Nota informativa & 372 & 87 \\
Reportajes & 30 & 7 \\
Editoriales & 17 & 4 \\
Desplegados & 6 & 1 \\
Secciones especializadas & 3 & 1 \\
Total & 428 & 100 \\
\hline
\end{tabular}

CUADRo 3. Distribución de unidades de análisis por periódico

\begin{tabular}{|c|c|c|c|c|c|}
\hline Periódico & Notas & Reportajes & Desplegados & $\begin{array}{c}\text { Secciones } \\
\text { especializadas }\end{array}$ & Editoriales \\
\hline Vanguardia & 155 & 3 & 5 & 0 & 8 \\
\hline Palabra & 144 & 17 & 1 & 1 & 9 \\
\hline El Diario & 72 & 10 & 0 & 2 & 0 \\
\hline
\end{tabular}

a) La agenda medioambiental de la prensa

Por otro lado y de acuerdo a los resultados obtenidos, se encontró que el tema sobre el medio ambiente, aunque es abordado sistemáticamente por la prensa local, no es un asunto que se encuentre de manera abundante entre las páginas de los diarios. Esto es, la problemática medioambiental presenta un "bajo perfil" en la agenda de los periódicos locales, ya que existen otros temas sociales que los periódicos privilegian tales como la inseguridad, la corrupción o las reformas fiscales.

Sin embargo, durante los ocho días del periodo analizado se encontró que la temática ambiental adquiró una mayor visibilidad, un "alto perfil" en la agenda, ya que se publicaron en promedio alrededor de 13 notas al día, entre los tres periódicos. Esto es, durante ocho días se ofrecieron a los lectores 105 unidades sobre el medio ambiente, las que correspondieron al $24 \%$ del total de la información difundida sobre el tema durante el periodo estudiado (cuadro 4), lo que pone de manifiesto que el incremento del perfil de la agenda depende, al igual que para otros temas, de las características del acontecimiento base de la información periodística, de la implicación de los distintos actores de la sociedad en el mismo acontecimiento $y$, por supuesto, del papel que juegan las fuentes "legitimadas", principalmente las gubernamentales y empresariales, que en muchos de los casos también determinan la agenda de los medios. 
Lo anterior en virtud de que la alta concentración de notas referidas en el cuadro 4 se debió, especialmente, a que en dichos días se generó, en diversos sectores de la población, una gran cantidad de declaraciones, presentaciones y reclamos sobre problemáticas ambientales muy variadas. Es decir, no existió ningún acontecimiento en particular que concentrara la atención de la prensa, sino que fue una gran producción de notas informativas acerca de distintos asuntos tales como: la problemática del agua, auditorias ambientales en Peñoles en la Región Laguna, la puesta en marcha de programas de educación ambiental en algunas escuelas de la localidad, algunos incendios en la sierra por la temporada, la muerte de mariposas monarcas a su paso por la ciudad, la presencia de basureros industriales clandestinos, y la falta de servicios primarios en algunas colonias de la periferia, entre otras.

Además, es importante aclarar que las notas informativas y reportajes que se publicaron durante estos ocho días, y que ofrecieron a la opinión pública reflexiones acerca de la problemática ambiental en la ciudad y la región, sólo presentaban una descripción del acontecimiento, pero, en ningún momento, ofrecían al lector argumentos para promover el análisis público de los asuntos del medio ambiente.

Los datos concentrados en el cuadro 4 también permiten deducir que, además de que los tópicos ambientales alcanzar un "alto perfil" durante un tiempo determinado, es evidente que un periódico, el Vanguardia, concentra cerca del $\mathbf{4 0}$ por ciento de la información emitida durante esos ocho días de mayor visibilidad del tema ambiental a través de sus diferentes tópicos.

Por otro lado, y al proceder a la identificación de los argumentos ambientales que conforman la agenda de los periódicos (cuadro 5), se encontró que el tópico con mayor recurrencia en la agenda es el referido a la "protección y manejo del agua" (32\%); en segundo lugar, el del "manejo de residuos sólidos y líquidos" (15\%), esto es, lo relacionado con la problemática de la basura en el municipio; el tercer problema visibilizado por la agenda de la prensa local es el que se refirió al "combate a la deforestación" (13\%); en cuarto lugar se encontraron las unidades de análisis sobre la "protección de la atmósfera" (11\%); posteriormente las notas que se refieren al "uso adecuado de los productos tóxicos" (8\%); el tema sobre "educación ambiental" (11\%) se hizo visible en sexto lugar y finalmente el tema sobre la "conservación de la biodiversidad" $(5 \%)$ es el último que tiene una presencia significativa en los periódicos del municipio.

De lo anterior, se puede destacar que la problemática del agua consigue el status de issue ya que a partir de su directa, inmediata y personal relación con los sujetos del sistema social, adquiere relevancia significativa para incluirlo de manera periódica en la agenda de los periódicos, lo que además, marca el principio de jerarquización de los mismos. 
CuADRO 4. Mayor concentración de notas por dia y por periódico*

\begin{tabular}{lclc}
\hline Fecha & Nüm. de notas & Periódico & Núm. de notas \\
\hline $13 / 03 / 02$ & 18 & Vanguardia & 11 \\
$21 / 02 / 02$ & 16 & El Diario & 12 \\
$18 / 03 / 02$ & 15 & Vanguardia & 10 \\
$31 / 03 / 02$ & 13 & Palabra & 7 \\
$14 / 02 / 02$ & 12 & Vanguardia & 7 \\
$25 / 03 / 02$ & 11 & Palabra & 6 \\
$07 / 03 / 02$ & 10 & Vanguardia & 7 \\
$18 / 02 / 02$ & 10 & Vanguardia & 4 \\
& 105 & & 64 \\
Total & & & \\
\hline
\end{tabular}

-Concentración de 10 o más notas por día.

b) La jerarquización de los temas ambientales

Con respecto a la jerarquía de la temática, entendida ésta como la importancia que se le da en la agenda periodística a cada uno de los problemas sobre el medio ambiente entre sí y con relación a otros, se puede decir, en primer lugar, que no existe un trato equitativo con respecto a otros temas ya que las unidades que refieren a los asuntos medioambientales sólo ocupan alrededor de $2 \%$ del espacio dedicado a la información en los periódicos estudiados debido, probablemente, a que se privilegian otros temas que sirven para acaparar la atención del público lector y consumidor final del producto informativo, tales como la inseguridad, la corrupción y las reformas fiscales, entre otros.

\section{CUADRO 5. Temática abordada}

\begin{tabular}{lcc}
\hline Tema & Nüm. de unidades & $\%$ \\
\hline Protección y manejo del agua & 137 & 32 \\
Manejo de sólidos y líquidos & 62 & 15 \\
Combate a la deforestación & 54 & 13 \\
Protección de la atmósfera & 47 & 11 \\
Uso adecuado de productos tóxicos & 34 & 8 \\
Educación ambiental & 25 & 6 \\
Conservación de la biodiversidad & 21 & 5 \\
Residuos peligrosos & 19 & 4 \\
Otros temas & 11 & 3 \\
Manejo del suelo & 6 & 1 \\
Combate a la desertificación y la sequía & 5 & 1 \\
Manejo de biotecnología, agricultura sustentable, & & \\
protección y manejo de océanos y manejo & 7 & 1 \\
de residuos radioactivos y sin tema & 428 & 100 \\
Total &
\end{tabular}


Así, sobre la jerarquía misma de algunos tópicos que conforman el tema ambiental, (cuadro 6) y su relación con las motivaciones de los periódicos para abordarlos, se encontró que de los 137 registros analizados sobre el tema "protección y manejo del agua", el periódico Palabra es la organización que le proporcionó mayor cobertura al mismo (46\%), seguido por los periódicos Vanguardia y El Diario.

Además, el ítem que se privilegió sobre la "protección y manejo del agua" fue el que se refiere al de "agua potable para todos", donde se agrupaban las demandas de los habitantes o vecinos de diferentes colonias de la localidad, así como también las declaraciones de diversos actores sociales como los directivos de Aguas de Saltillo (AgSal), Aguas de Barcelona, la presidencia municipal, las cámaras empresariales, y los representantes religiosos. Notas relacionadas siempre con las denuncias o quejas sobre la falta de agua o servicio deficiente en distintos sectores de la población, lo que pone de manifiesto, nuevamente, que la noticiabilidad de un acontecimiento está relacionada con la "legitimación" de las fuentes y que éstas son las que finalmente institucionalizan socialmente los discursos informativos (Rodrigo, 1989: 120).

Por otra parte, el asunto sobre el pago de agua a los Estados Unidos fue otro de los problemas abordados por la prensa durante el periodo de estudio, especialmente a través de entrevistas a ejidatarios, no sólo locales, sino de los tres estados que conforman la región: Nuevo León, Tamaulipas y Coahuila.

CUADRO 6. Relación medios y principales problemas del medio ambiente

\begin{tabular}{lcccc}
\hline Tema & Vanguardia & Palabra & El Diario & Total $U$ \\
\hline $\begin{array}{l}\text { Protección y manejo } \\
\text { de aguas dulces }\end{array}$ & $38 \%(51)$ & $46 \%(63)$ & $17 \%(23)$ & 137 \\
$\begin{array}{l}\text { Manejo de residuos } \\
\text { sólidos y liquidos }\end{array}$ & $54 \%(34)$ & $32 \%(20)$ & $13 \%(8)$ & 62 \\
Combate a la deforestación & $35 \%(19)$ & $23 \%(12)$ & $42 \%(23)$ & 54 \\
$\begin{array}{l}\text { Protección de la atmósfera } \\
\text { Uso adecuado de productos }\end{array}$ & $45 \%(21)$ & $40 \%(19)$ & $15 \%(7)$ & 47 \\
tóxicos & $40 \%(14)$ & $45 \%(15)$ & $15 \%(5)$ & 34 \\
Educación ambiental & $40 \%(10)$ & $40 \%(10)$ & $20 \%(5)$ & 25 \\
\hline
\end{tabular}

Otro ítem referido a la problemática del agua fue el del "tratamiento de aguas negras". Acerca de este asunto se observó un detallado seguimiento sobre el proceso de licitación de la planta tratadora de aguas que se planea construir en la localidad, en donde las principales fuentes de la información fueron los funcionarios de la presidencia municipal, el comité ciudadano establecido para tal fin y los empresarios de la localidad. Lo que pone de manifiesto que la prensa puede desempeñar un papel 
importante en temas como éste a través de la puesta en la agenda del medio de la discusión generada sobre los asuntos que conciernen a la preservación del medio ambiente.

Sobre el tópico que se refiere al "manejo de residuos sólidos y líquidos" (basura) (cuadro 6), del cual se contó en total con 62 registros, la distribución de las notas por periódico se presentó de manera distinta al caso del agua, ya que fue Vanguardia (54\%) quien se ocupó principalmente del asunto, seguido de los periódicos Palabra (32\%) y finalmente del informativo El Diario (13\%).

Respecto al ítem más sobresaliente sobre el manejo de la basura, se encontró que fue el referido al "tratamiento adecuado de los residuos", en donde se abordaron problemáticas como el establecimiento ilegal de rellenos sanitarios, tiraderos clandestinos de basura, así como un alto confinamiento de basura en arroyos y baldíos. Además, es importante destacar que este tema es el más frecuentemente editorializado por el periódico Vanguardia, siempre reflexionando acerca de la falta de conciencia o educación de la población para un manejo adecuado de la basura.

Si bien es cierto que fue el periódico Vanguardia el que manejó la gran mayoría de notas sobre la basura, es importante señalar que, en el periódico El Diario, se encontró que todas las notas publicadas sobre el manejo de los residuos sólidos se refirieron a este ítem, el cual se abordaba en una sección denominada "Inspector Urbano: el recorrido de la semana", en la que se pone de manifiesto, desde la perspectiva de los vecinos, las distintas problemáticas que viven los habitantes de diferentes colonias.

Sobre el tópico clasificado en tercer lugar, y que se refiere al "combate a la deforestación" (cuadro 6) se registraron 54 notas, y la distribución por periódicos fue $E l$ Diario, 42\%; Vanguardia, 35\% y Palabra el 23 por ciento.

Es importante destacar que gran número de notas de este tema fueron manejadas por el periódico El Diario y que esta preferencia tiene relación directa con los intereses de la familia propietaria del mismo, ya que algunos de sus integrantes han desempeñado funciones en la política local. El dueño del periódico tiene la profesión de agrónomo, ha sido presidente municipal, Secretario de Agricultura en el estado y actualmente, uno de sus hijos dirige uno de los complejos de ecoturismo más importantes en la Sierra de Arteaga, zona de alto riesgo en incendios. Además, uno de los hermanos [del dueño] en la administración estatal actual es el Secretario de Fomento Agropecuario, lo que tiene una relación directa con el subtema manejado más frecuentemente y que se refiere al "control de incendios". Por otra parte, es de llamar la atención que sólo se publicaron dos notas sobre la política forestal, lo que también lleva a pensar que a la prensa y, por supuesto, a los funcionarios gubernamentales, no les interesa proporcionar otro tipo de información que no 
sea aquélla que directamente tenga una relación con un acontecimiento que alcance niveles altos de espectacularización y que ayude a reforzar la opinión pública sobre la imagen del trabajo del mismo gobierno.

Sobre la "protección de la atmósfera" (cuadro 6) se localizaron 47 unidades. La distribución de dichas notas en cada uno de los periódicos es como sigue: el Vanguardia, 45\%; Palabra, $40 \%$ y El Diario, $15 \%$. El ítem que mayoritariamente conformó la agenda de la prensa es el que se refiere a "las alternativas para combatir la contaminación atmosférica" y es precisamente el periódico Vanguardia el que más notas ofrece a los lectores sobre este asunto, proporcionando un seguimiento detallado acerca del asunto de la conversión del energético utilizado en la producción de ladrillo, actividad económica importante en la localidad, y que desde su establecimiento fue uno de los principales contaminantes atmosféricos a partir de la emisión de gases tóxicos producto de la quema de llantas. Cabe mencionar que también la mayoria de las notas del Palabra abordan dicho ítem y en menor medida el periódico El Diario.

Con respecto a "el uso adecuado de productos tóxicos" (cuadro 6), se identificaron 34 notas. El periódico Palabra aborda mayormente dicho tópico (45\%), en segundo lugar el periódico Vanguardia (40\%) y, por último, el periódico $\mathrm{El}$ Diario (15 por ciento).

Los ítems que se abordaron con mayor frecuencia en el periódico Palabra son los relacionados con accidentes y explosiones producto del mal manejo de los productos tóxicos. Acontecimientos que ofrecen al reportero y al medio una gran cantidad de elementos para su espectacularización.

Sobre el tópico de "educación ambiental" (cuadro 6) se analizaron 25 notas, las cuales se concentraron en los periódicos Vanguardia y Palabra en un $40 \%$ respectivamente y en el periódico El Diario sólo el $20 \%$. Estas notas correspondían a anuncios, discursos del gobernador del tema e inauguraciones de cursos o talleres educativos para niños, entre otros. Pero en ninguno de los periódicos se maneja información amplia o recomendaciones para mejorar la actuación social acerca del medio ambiente, ni por supuesto, cuestionamientos sobre las políticas y/o estrategias que se siguen en la educación ambiental en la región.

Los datos anteriores ponen de manifiesto dos cosas: por un lado, que algunos tópicos alcanzan un "alto perfil" en un periódico con respecto a otro; pero, también, que algunos de los ítems que conforman los tópicos identificados en este análisis adquieren un "alto perfil" con respecto a los otros ítems que conforman el tópico en cuestión.

Desde este punto de vista, resulta importante destacar cómo los distintos medios impresos le dan a cada tema o problemática medioambiental una importancia distinta, determinada, probablemente, por las mediaciones institucionales y personales que se encuentran en la naturaleza de la misma organización periodística y en los equipos de profesionales que 
participan en ella, como se ejemplifica en el periódico $E l$ Diario y que, por lo tanto, intervienen en la conformación de la agenda que se pone en circulación cada día, pues desde sus propias percepciones determinan si son acontecimientos periodísticamente interesantes.

Por otro lado, y para entender mejor la jerarquización de los temas ambientales en la agenda de la prensa de Saltillo, es importante contar con datos que refieran la procedencia de las noticias, el lugar donde se generan, así como, las fuentes utilizadas para este tipo de información y finalmente la ubicación que ocupan en el diseño final del periódico.

Así, la procedencia de la nota refiere al lugar donde se dio a conocer la información que se publica. De acuerdo a los datos obtenidos (cuadros 7), $83 \%$ de la información procedió de las dos regiones más densamente pobladas; esto es, de la regiones Sureste y Laguna; $4 \%$ del Distrito Federal, y 3\% de la Región Frontera del estado. Por otra parte, el lugar donde se generaron las noticias, y que se refiere al sitio del cual se habla o se hace referencia en la información analizada, de acuerdo a los datos obtenidos, se registró de la siguiente manera (cuadro 8): $84 \%$ correspondía a información generada en seis de los 38 municipios que conforman el estado: Saltillo, Torreón, Ramos Arizpe, Arteaga, Piedras Negras y Acuña, espacios geográficos que concentran al mayor número de habitantes de la entidad y, por ende, la actividad económica y cultural, pero además, las problemáticas ambientales.

Para la prensa local incluida en este estudio, las fuentes informativas fueron principalmente los representantes de las dependencias gubernamentales (cuadro 9), tanto del nivel estatal como municipal. Pero, es importante destacar, además, que $28 \%$ de las fuentes están conformadas por los diferentes grupos sociales que representan una destacada actividad ciudadana con respecto al medio ambiente, tales como grupos ecologistas, asociaciones de vecinos, testigos de eventos contaminantes, usuarios de servicios, vecinos de colonias afectadas por la violación a las recomendaciones sobre el uso de los recursos del medio ambiente, entre otros.

Además, la página en la que aparecen mayoritariamente las notas en este estudio (79\%) es en la Sección B de los periódicos, esto es, en la sección donde se aborda la problemática de la localidad. El $29 \%$ restante se encuentra distribuido entre las diferentes secciones de los periódicos estudiados: internacional, nacional o social.

Por otra parte, debemos considerar que, si bien es cierto que la agenda de los medios se encuentra conformada por los temas de actualidad presentes durante un periodo específico, cada uno de los tópicos sobre el medio ambiente está construido por una serie de ítems que también influyen en la jerarquización de las temáticas y que finalmente se encuentran íntimamente relacionados con la agenda de los "otros" actores sociales que forman parte activa en el establecimiento de la misma: los informantes/fuentes y los lectores. 
CUADRo 7. Procedencia de las noticias

\begin{tabular}{ll}
\hline Lugar & $\%$ \\
\hline Saltillo & 67 \\
Torreón & 7 \\
Ramos Arizpe & 7 \\
Distrito Federal & 4 \\
Arteaga & 2 \\
Piedras Negras & 2 \\
Acuña & 1 \\
Resto de los municipios y estados de la República & 10 \\
\hline
\end{tabular}

CUADRO 8. Lugar de generación de las noticias

\begin{tabular}{lc}
\hline Lugar & $\%$ \\
\hline Saltillo & 60 \\
Torreón & 8 \\
Ramos Arizpe & 7 \\
Arteaga & 4 \\
Sabinas & 3 \\
Piedras Negras & 2 \\
Resto de los municipios y estados de la República & 16 \\
Total & 100 \\
\hline
\end{tabular}

CUADRO 9. Principales sectores/fuentes

\begin{tabular}{lc}
\hline Sector & $\%$ \\
\hline Gobierno estatal y municipal & 45 \\
Sociedad civil & 28 \\
Gobierno federal & 10 \\
Sector privado municipal & 7 \\
Medios de comunicación & \\
$\quad$ Editorialistas) & 4 \\
Sindicatos y organizaciones nacionales, estatales y municipales & 2 \\
Partidos políticos & 2 \\
Sector privado nacional y estatal, organismos religiosos y universidades & 2 \\
y centros de investigación & 2 \\
Total & 100 \\
\hline
\end{tabular}




\section{Reflexiones finales}

Finalmente, ¿qué respuesta/s se pueden dar a la pregunta sobre si los temas ambientales forman parte de la agenda de los periódicos que circulan en el municipio de Saltillo? De acuerdo a los datos obtenidos y presentados en líneas anteriores, se pueden enunciar las siguientes.

En primer lugar, si bien es cierto que la prensa incluye aspectos sobre la problemática del entorno natural, ésta presenta un "bajo perfil" en la agenda de los periódicos con respecto a otros temas como la seguridad pública, la corrupción o las reformas fiscales ya que sólo ocupa en promedio $2 \%$ del espacio de los mismos. Sin embargo, es un tema que se mantiene de manera discreta, sistemática y permanente a través de notas informativas que pretenden, en algunos casos, convertir en espectaculares los acontecimientos que se producen en la sociedad (primordialmente, las declaraciones gubernamentales o los reclamos de la sociedad civil a las autoridades municipales sobre la falta de agua y/o el deficiente manejo de la basura).

Aunque existen algunos periódicos como Palabra y El Diario que ofrecen a sus lectores reportajes y secciones especializadas acerca de algunas temáticas, hay muy poca reflexión o trabajo de periodismo de investigación que ofrezca a los lectores elementos informativos que permitan reconstruir el conocimiento que sobre los temas o problemáticas del medio ambiente, local y regional, se ponen de manifiesto en la actualidad y con esto incidir en la construcción de una conciencia ciudadana sobre las cuestiones ambientales.

También es importante comentar que este tema, como ha sido observado por otros (Dunlap, 2001 y Hansen, 1991), puede elevar su presencia en la prensa, de acuerdo a los acontecimientos que se presenten en la sociedad ya sea determinados por los elementos biosociales o por las acciones de las fuentes generadoras de la información. Como es el caso de los ocho días que lograron elevar su presencia en la agenda, al incrementar entre el 13 y el $27 \%$ el número de notas presentadas, en donde el periódico Vanguardia es el que en mayor medida incrementó la información.

Así, la revisión de los datos sobre el tema permitió distinguir los acontecimientos que mayor importancia issue alcanzaron en la prensa local, como son: la protección y el manejo del agua, el manejo de sólidos y líquidos, el combate a la deforestación, la protección de la atmósfera, el uso adecuado de productos tóxicos y la educación ambiental. Considerados temas centrales ya que manifiestaban una trascendencia social, sobre todo para los sujetos protagonistas en las mismas unidades, ya sea el gobierno o la sociedad civil. Al igual que por la importancia de la temática para el desarrollo sustentable de la sociedad saltillense.

Además, porque contienen, en esencia, los elementos que en todo acontecimiento se pueden identificar, como es la variación del sistema, 
que se manifiesta no solamente con la relación del tiempo, en la que alcanzaron su noticiabilidad los diferentes temas, puesto que a lo largo del tiempo se les fue manejando con distintas variaciones como fueron las declaraciones de distintos sectores o las evidencias de problemas administrativos que al mismo tiempo sirvieron para espectacularizar, tal como se manejó el caso de la constitución de la empresa paramunicipal de aguas (AgSal), en donde a partir de aspectos como la nacionalidad o sueldos de sus funcionarios y una sola versión de dichas variantes la nota se trabajó sin llegar a dar una explicación completa del acontecimiento. Todo eso con el fin de producir sensaciones, emociones o impresiones con las noticias o sucesos que ponen en circulación, lo que acarrea un grave error en el momento de hablar de temas ambientales ya que no se alcanza a percibir la magnitud de los acontecimientos.

Otro elemento importante identificado es el alto grado de implicación de los sujetos en la temática abordada; aquí es importante recordar que la problemática del medio ambiente, por lo regular tendrá una mayor implicación en los sujetos de una microrregión, cuando los problemas se encuentren afectándolos directamente; por tal motivo, la implicación de los sujetos en este tipo de información será un elemento que matice la importancia de la información emitida.

Por otro lado, a partir de los datos aportados a través del análisis de contenido, se puede distinguir que cada uno de los periódicos responde a una tendencia o preferencia en el manejo de los "tópicos" sobre el medio ambiente, lo que pone de manifiesto que dependiendo de las características e intereses del mismo medio le darán una mayor o menor importancia. Así, Palabra privilegió el problema del agua, mientras que el periódico Vanguardia le dio un mayor énfasis a la problemática del manejo de los residuos sólidos (basura) y, por último, El Diario manifiesta una clara inclinación a ofrecer información del combate a la deforestación (incendios forestales).

Sin embargo, si reflexionamos que si los acontecimientos enmarcados en los medios de comunicación definen a la sociedad, la información proporcionada a través de las unidades analizadas en la prensa saltillense, nos confirma una sociedad que sigue pensando que la responsabilidad de resolver la problemática del medio ambiente sólo le corresponde a las instancias gubernamentales, sin reconocer que desde la misma sociedad civil se pueden construir propuestas alternativas que contribuyan de manera directa a la solución de los problemas ambientales de la vida diaria, y dejar de lado la idea de adoptar formas de actuar diseñadas desde fuera de las propias comunidades.

Existen otros acontecimientos que, aunque son abordados por la prensa no alcanzan a tener un reconocimiento mayor en relación con los otros y por lo tanto no logran una permanencia en la agenda de los medios. En este tipo se clasifican los temas que pusieron de manifiesto asuntos como, 
el manejo de residuos peligrosos, el manejo del suelo, el combate a la desertificación y la sequía, manejo de biotecnología, la agricultura sustentable, y la protección y manejo de océanos y de residuos radioactivos. El tema sobre el desarrollo sustentable en las regiones montañosas es el único que no fue abordado en ninguna de las unidades de análisis estudiadas, aunque en algún momento podría tener importancia local por contar en la región con zonas agrícolas en las montañas cercanas a la ciudad.

Por último, es importante plantear que, si bien los problemas ambientales son abordados por los periódicos del municipio, éstos no alcanzan a contextualizar en toda su dimensión la realidad ambiental de la localidad, en principio porque son sólo algunos los tópicos que se abordan, pero también porque los contenidos de la información que se maneja se encuentran a un nivel básico, no se profundiza en los temas y se dibujan escenarios a medias. Sin embargo, este primer acercamiento a la agenda ambiental de los asuntos del medio ambiente, proporciona indicadores importantes para quienes toman decisiones acerca de los aspectos ambientales, pues permite un acercamiento al imaginario que tiene la prensa del asunto ambiental y que en un proceso cognitivo de largo plazo puede ir construyendo el imaginario social del tema; en segundo lugar, porque estos datos ayudarían a fundamentar la implementación de estrategias comunicativas acerca del tema ambiental a distintos niveles de incidencia.

\section{Bibliografia}

Cienfuegos Salgado, David (2003), "Reflexiones ambientales del siglo que pasó", en Revista Lex, tercera época, año VIII, octubre, pp. 10-23.

Castells, Manuel (2001), La era de la información. Economía, sociedad y cultura, vol. II, $3^{a}$. ed., Siglo XXI, México.

Dunlap, Riley, E., Frederick H. Buttel, et al. (2002) Sociological theory and the environment. Classical foundations, Contemporary Insights, Rowman \& Littlefield Publishers, Inc., Boston.

- y Rik Scarce (1991), "The polls-poll trens. Environmental problems and protection", en Public Opinion Quarterly, vol. 55, p. 651.

Esteinou, Javier (2001), "Ecología y medios de comunicación en México", en Cuadernos del Ticom, Nueva Época, UAM-Xochimilco, México.

Fregoso, Gilberto (1993), "La prensa en Guadalajara: cuatro versiones de una tragedia", en Comunicación y Sociedad, núms. 16-17, CEIc/Universidad de Guadalajara, Guadalajara.

Hansen A, (1991), "The media and the social construccion of the enviroment", en Media, Culture and Society, 13 (4), pp. 443-458.

Lozano Rendón, José Carlos (1994), "Hacia la reconsideración del análisis de contenido en la investigación de los mensajes comunicacionales", en Cecilia Cervantes Barba y Enrique E. Sánchez Ruiz (coords.), Investigar la comunicación: propuestas iberoamericanas, UdeG/CEIC/ALAIC, México, pp. 135-157. 
McCombs Maxwell E. y Donald L. Shaw (1993), "The evolution of Agenda Setting Research: twenty-five years in the marketplace of ideas", en Journal of Communication, 43 (2), primavera.

Rodrigo Alsina, Miquel (1989), La construcción de la noticia, Paidós, México.

Saperas, Enric (1987), Los efectos cognitivos de la comunicación de masas. Las recientes investigaciones en torno a los efectos de la comunicación de masas: 1970-1986, Ariel, Barcelona.

Serrano, Manuel Martín (1993), Teoría de la comunicación: epistemología y análisis de la referencia, UNAM, México.

Sato, Michèle y José Eduardo dos Santos (1997), Sinopsis de la Agenda 21, SEMARNAT/PNUD, México.

Wolf, Mauro (1991), La investigación de la comunicación de masas: crítica y perspectivas, Paidós, México.

Yescas Lagunas, Germán (1999-2000), "La difusión ambiental a través de audiovisuales", en Razón y Palabra [revista electrónica] 16 (4). Disponible en: http://www.razónypalabra.org.mx. 